\title{
TOMOGRAFÍA EN PANCREATITIS, ¿ALGO HA CAMBIADO?
}

Rafael Riveros Dueñas, M.D. *, Raúl Guevara Castro, M.D. **

\section{Resumen}

La pancreatitis aguda severa se define como aquella asociada a falla orgánica y/o complicaciones multisistémicas. Con el fin de comparar la capacidad del puntaje de APACHE II y la tomografía dinámica (Índice Balthazar Ranson), se llevó a cabo un estudio en el que participaron los pacientes con diagnóstico de pancreatitis aguda, entre marzo de 1999 y junio de 2000. Del total de pacientes, $60 \%$ fueron llevados a cirugía. El grado de correlación del APACHE II con la severidad del cuadro clínico y la morbimortalidad posterior es excelente, mientras que la tomografía dinámica, realizada en las primeras $\mathbf{7 2}$ horas no fue un buen marcador pronóstico y, por lo tanto, su utilidad en esta situación es cuestionable.

\section{Introducción}

El consenso internacional de Atlanta define la pancreatitis aguda severa como aquella asociada a falla orgánica y/o con complicaciones locales, como necrosis, absceso o pseudoquiste; caracterizada por tres o más criterios de Ranson o un puntaje de ocho o mayor del APACHE II (evaluación fisiológica aguda y crónica de enfermedad). ${ }^{1}$

Múltiples estudios muestran diferencias en al morbilidad, mortalidad y la necesidad de cirugía, según si la categorización de los pacientes considera la puntuación de APACHE II o los criterios de Ramson. Aunque ello confirma la utilidad de los dos métodos, pese a una identificación temprana de la pancreatitis aguda (PA), la mortalidad por esta causa, en los pacientes que desarrollan falla orgánica múltiple en la primera semana, no ha mostrado un descenso significativo en los dos últimos decenios.

Diferentes marcadores séricos se han estudiado para tener un pronóstico más exacto del curso de la

\footnotetext{
* Cirujano General, Departamento Quirúrgico, Hospital de San José. Bogotá, D.C.

** Residente, Departamento Quirúrgico, Hospital de San José, Bogotá, D.C.
}

pancreatitis: IL-6, IL-10, antagonista del receptor para IL-1, factor de crecimiento del hepatocito, productos de degeneración del colágeno y la IL-8. ${ }^{2} \mathrm{La}$ mayoría de estos identifican mejor y más temprano pacientes con posibilidad de complicaciones sistémicas severas, sin necesidad de realizar estudios costosos e invasivos. Faltan más trabajos que confirmen estos resultados para que se popularicen y universalicen estas pruebas, como lo que sucedió con la tomografía abdominal en los años ochenta.

En estas condiciones, y dada su capacidad de mostrar necrosis, la tomografía computarizada dinámica (TAC dinámica) sigue siendo una herramienta útil en la identificación de los pacientes con pancreatitis aguda severa. No obstante, ha sido debatida la utilidad de esta tomografía para predecir complicaciones sistémicas severas, que generalmente desencadenan la muerte en las dos primeras semanas de instaurada la enfermedad.

Cuando se inició el uso de la tomografía computarizada abdominal para el diagnóstico de la PA, esta técnica se realizaba sin contraste, por lo que no se demostraba la necrosis. ${ }^{3}$ Basados en las observa- 
ciones de Kivisaari, algunos autores colocaban infusiones de medio de contraste sin lograr documentar necrosis; los trabajos de Friedman y Modder en los que se combinaban bolos intravenosos rápidos de medio de contraste con cortes abdominales tomográficos, lograron detectar áreas hipoperfundidas que en estudios histológicos demostraron corresponder a focos de necrosis.

La tomografía computarizada dinámica con infusión intravenosa rápida (TAC dinámica-CECT), logró diferenciar en forma adecuada las áreas de edema de las de necrosis, reportando una sensibilidad de $87 \%$ y una especificidad de $100 \%$. Para hacer que esta prueba tenga una valoración objetiva se toma como valor de corte una densidad menor de $40 \mathrm{UH}$ del parénquima pancreático, densidad que puede ser variable por el volumen sanguíneo, el flujo sanguíneo pancreático, la difusión del medio de contraste o los cambios anatómicos del paciente.

Otra forma de valorar de manera rápida las placas tomográficas, sin la necesidad de marcar previamente con el cursor el área de estudio, es comparar la densidad del parénquima pancreático con la de la aorta que debe tener medio de contraste en su interior (índice páncreas: aorta). La necrosis se presenta como una zona hipodensa, debido a la reducción del flujo sanguíneo pancreático y al daño del flujo capilar. Este daño del flujo capilar es secundario a la alteración del endotelio microvascular, lo que da como resultado trombosis de los vasos aferentes y esta condición altera la perfusión del medio de contraste. ${ }^{4}$

Ranson y Balthazar ${ }^{5}$ clasificaron los hallazgos de la TAC en la PA así: A: normal; B: aumento pancreático; $\mathrm{C}$ : inflamación confinada al páncreas y grasa peripancreática; D: colección líquida peripancreática; E: dos o más colecciones líquidas. Posteriormente, Balthazar, Ranson y colaboradores ${ }^{6}$ adicionaron a esta clasificación una graduación de la necrosis, formando así el índice combinado de severidad tomográfico. Este índice de severidad se obtiene sumando las dos graduaciones. Pacientes que tienen un índice de severidad de 0 a 1 no tienen mortalidad o morbilidad, índice de 2 no presentan mor- talidad y solo $4 \%$ de morbilidad, en contraste con pacientes que tienen un índice de severidad de 7 a 10 donde existe una mortalidad de $17 \%$ y una morbilidad de $92 \%$.

El trabajo de London y colaboradores ${ }^{7}$ muestra que el sitio y la extensión de la necrosis no se correlacionan con la severidad de la enfermedad, ni señalan qué pacientes se benefician de una intervención quirúrgica. Sólo se le da importancia a la TAC dinámica para el planeamiento quirúrgico y para obtener información previa a la laparotomía.

La TAC dinámica es un examen costoso y su uso en todos los pacientes con pancreatitis leve es cuestionable..$^{4,7,8-13}$ Está indicado en pacientes con enfermedad severa, en aquellos que evidencian deterioro clínico o en quienes tienen más de tres criterios de Ranson. $^{10}$

Otro método para la identificación de pacientes con PA es la evaluación fisiológica aguda y del estado de salud crónico (APACHE II). Este método utiliza los valores más bajos de 12 mediciones fisiológicas, edad y estado de salud previa, mostrando un dato objetivo y confiable de la severidad de una enfermedad. Las variables fisiológicas son: temperatura, presión arterial media, pH arterial, sodio sérico, potasio sérico, creatinina sérica, hematocrito, leucocitos y la escala de coma de Glasgow. Cada variable tiene un puntaje de 04, dependiendo de su valor. ${ }^{14}$

En el trabajo de Marvin y McMahon, ${ }^{15} \mathrm{y}$ posteriormente en los de Healt e Imrie, ${ }^{16}$ se mostró que el APACHE II aportaba una discriminación objetiva entre los episodios no complicados, complicados y fatales de la PA.

Las ventajas del APACHE II sobre los sistemas pronósticos múltiples comprenden varios aspectos. Por una parte, este permite una determinación objetiva de la severidad de la PA, pocas horas después de la admisión, identificando tempranamente los pacientes que requieren terapia intensiva. Se basa en el uso de laboratorios de rutina, disponibles las 24 horas del día, poco costosos y de ejecución rápida. Además, hace posible la reevaluación diaria, dando así infor- 
mación para establecer el estado del paciente, la respuesta al tratamiento instaurado y la necesidad de otros.

Por otra parte, los puntajes iniciales en pacientes con PAsevera aumentan en las primeras 48 horas (promedio de tres puntos), pero decrecen en la leve (media de un punto). ${ }^{15}$ A ello se suma la posibilidad de manejar un puntaje universal para todas las patologías sistémicas, desechando así el uso de múltiples sistemas de puntaje de severidad.

El APACHE II se desempeñó mejor que los criterios pronósticos múltiples para la pancreatitis de origen biliar, pero no para la pancreatitis alcohólica donde los criterios de Ranson fueron mejores.

Las principales fallas en el APACHE II cubren una serie de situaciones que se enumeran enseguida: complejidad del cálculo; el corte ideal en los valores para PA debe ser revisado; los puntajes adecuados para la admisión de los pacientes deben ser diferentes para el seguimiento de los mismos; el simple corte en APA$\mathrm{CHE}>9$, propuesto por McMahon, necesita ser validado por otros estudios; el método es influenciado por la tardanza en la presentación de los síntomas y por el tipo de resucitación empleada; asigna demasiado puntaje para la edad (seis puntos por ser mayor de 75 años), dado que la edad como factor de riesgo para pancreatitis es controversial. Por último, no existen estudios en nuestro medio que comparen la capacidad de predecir qué pacientes presentarán un curso fatal o con mayor número de complicaciones entre el puntaje del APACHE II y la tomografía dinámica (índice Balthazar Ranson).

\section{Materiales y métodos}

Ingresaron al estudio todos los pacientes a quienes se diagnosticó PA, con criterio clínico, paraclínico e imagenológico, tras ser admitidos al Servicio de Cirugía del Hospital de San José, en Bogotá, entre el primero de marzo de 1999 y el 30 de junio de 2000.

Fueron excluidos los enfermos que ingresaron con un cuadro clínico superior a 48 horas, así como quienes fueron manejados en otra institución por más de
24 horas o aquellos a los que no se les realizó TAC dinámica.

Se recopilaron todas las TAC dinámicas realizadas al ingreso de los pacientes y se calculó el APACHE II, como lo ordena el protocolo existente en esta institución. Las tomografías fueron leídas por un radiólogo y un cirujano, junto con un formato que contiene un cuestionario, que evalúa la calidad y el diagnóstico tomográfico sin aportar más datos clínicos que el de PA.

La calidad fue valorada de acuerdo a los cinco criterios siguientes, y se consideraron adecuados los que obtuvieron más de 8 puntos. Dinámica o no $=5$ puntos; cortes finos $=3$ puntos; opacificación duodenal $=1$ punto; ventana adecuada $=1$ punto $\mathrm{y}$ $\sin$ artificios de movimiento $=1$ punto

El criterio de lectura fue la clasificación de Balthazar; el porcentaje de necrosis y otros hallazgos adicionales. Luego de cruzar los datos, se calculó el índice de severidad Balthazar-Ranson.

En una fase ulterior, el resultado de esta lectura y del APACHE II de ingreso se correlacionó con la evolución clínica final de cada paciente, dada por la morbilidad, la necesidad de ingresar a la unidad de cuidados intensivos, el requerir cirugía de urgencia, los hallazgos intraoperatorios y la mortalidad para determinar su valor pronóstico.

\section{Resultados}

Ingresaron al Servicio de Cirugía del Hospital de San José entre los meses de mayo de 1999 y junio de 2000, 49 pacientes a los que se diagnosticó PA. De estos se excluyeron 19 pacientes por presentar sintomatología mayor de 48 horas y 5 a los que no se les realizó tomografía dinámica.La etiología más frecuente es la de origen biliar con 18 pacientes, seguida por la idiopática con 4 , la alcohólica con 2 y la post-colangiografía retrógrada con 1 (Figura 1).

La severidad de la PA, discriminada como leve o severa según la clasificación del consenso de Atlanta, fue de $68 \%$ de pancreatitis severas y $32 \%$ leves. 


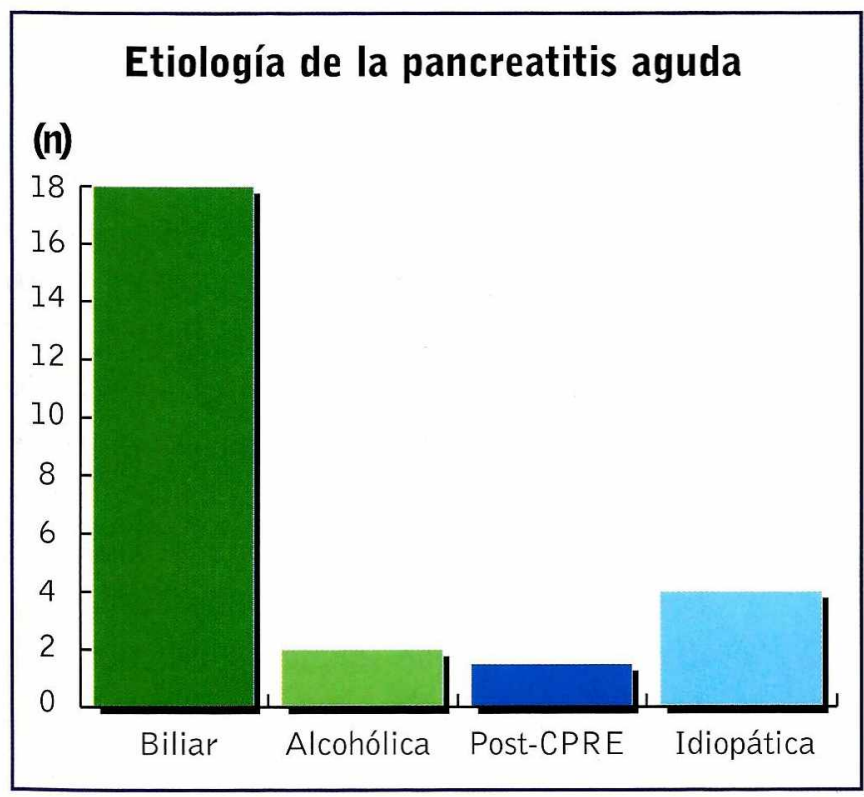

Figura 1

Las complicaciones sistémicas de los pacientes fueron: síndrome de dificultad respiratoria del adulto (30\%), falla orgánica múltiple (20\%), insuficiencia renal aguda (10\%), isquemia mesentérica $(10 \%)$ y sinusitis por sonda (10\%).

Las complicaciones locales correspondieron, en orden de importancia a sangrado intraabdominal (30\%), pseudoquiste (20\%), absceso (10\%) y la colección líquida aguda (10\%) (Figura 2).

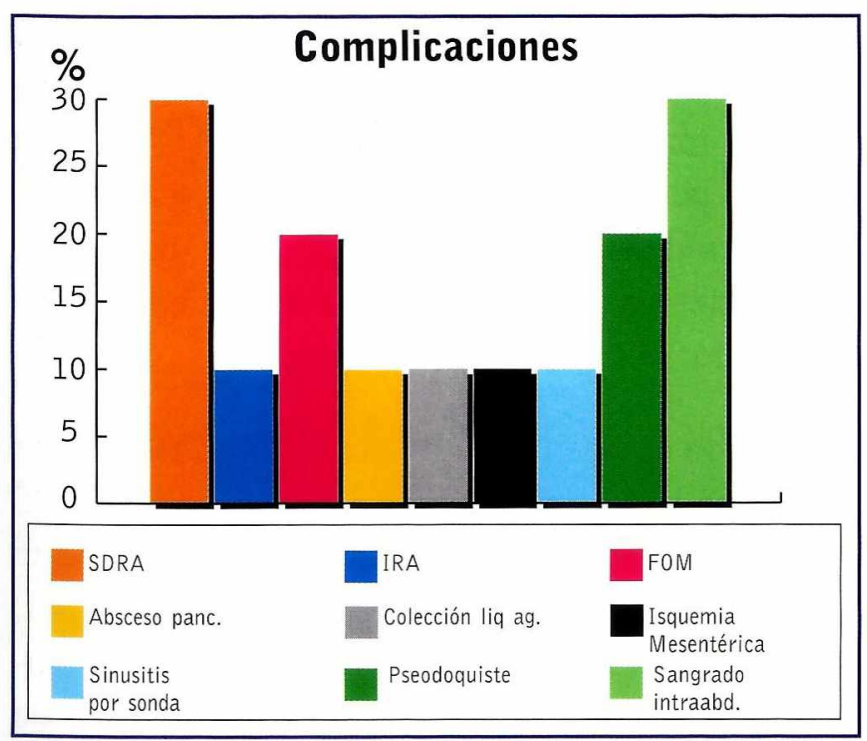

Figura 2
La indicación quirúrgica de los pacientes que fueron llevados a cirugía (60\%) fue: colecistectomía programada (26\%), deterioro clínico (13\%), abdomen agudo quirúrgico (13\%), colangitis (4\%) y patología biliar aguda (4\%) (Figura 3).

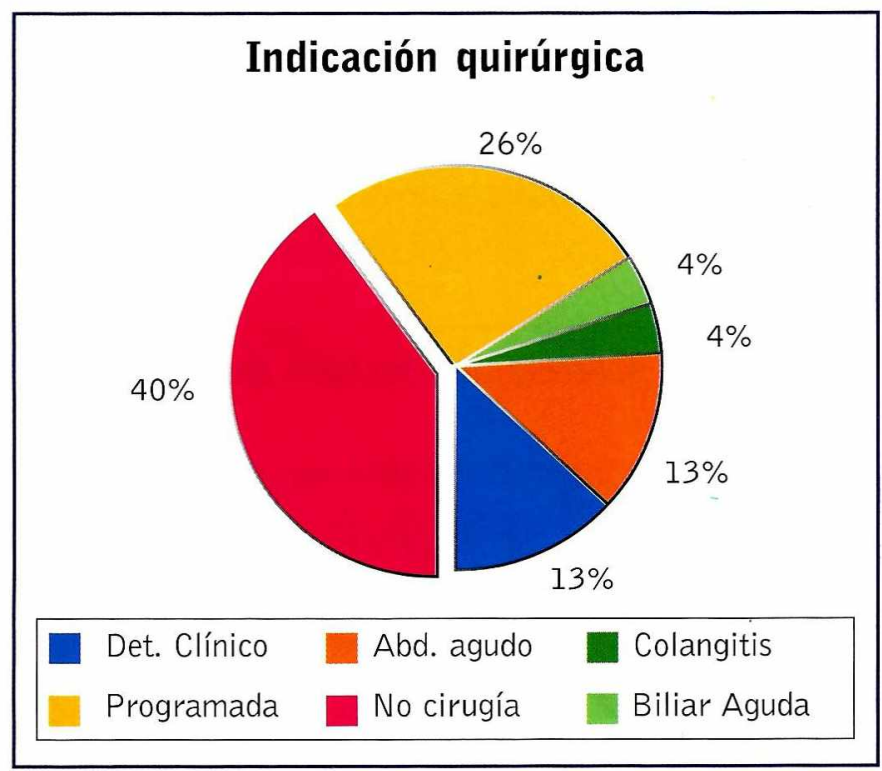

Figura 3

Los hallazgos quirúrgicos más comunes fueron: ascítis $(60 \%)$, colelitiasis $(50 \%)$, esteatonecrosis (40\%), necrosis mayor de 50\% (30\%), colecistitis aguda (30\%), necrosis $30-50 \%$ (10\%) y colangitis (5\%) (Figura 4).

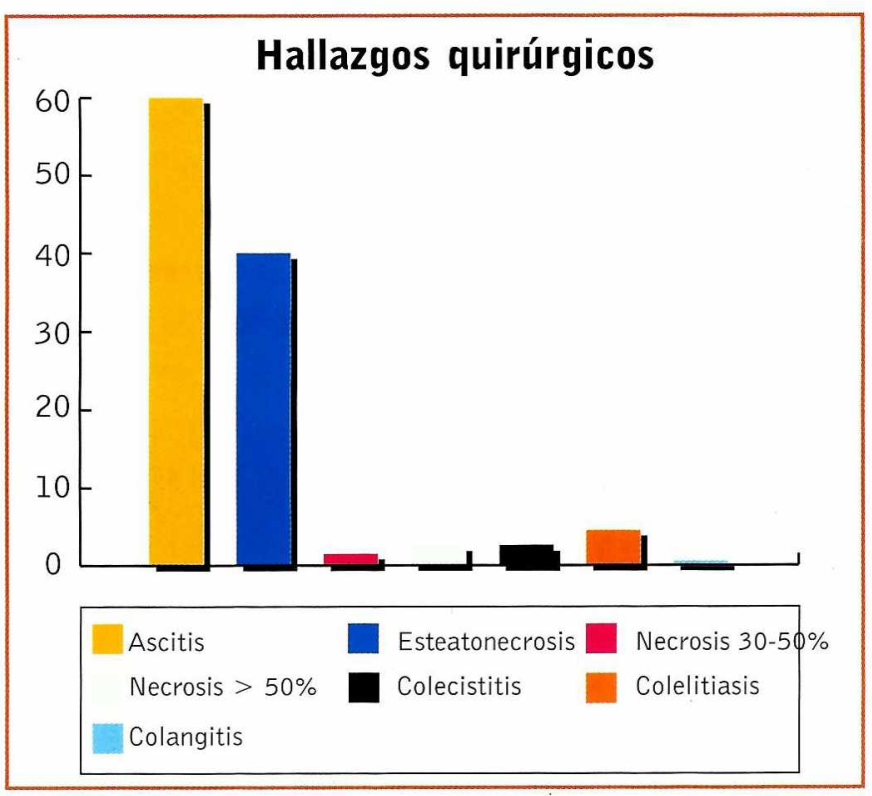

Figura 4 
La evolución de los pacientes clasificados mediante TAC y APACHE II como pancreatitis severa y leve respectivamente mostró una adecuada correlación de necesidad de Unidad de Cuidados Intensivos $(\mathrm{P}<0,004)$, morbilidad $(\mathrm{P}<0,0002)$, mortalidad $(\mathrm{P}<0,17)$ y necesidad de cirugía de urgencia en los

La correlación en la lectura de las tomografías por parte del radiólogo y el cirujano fue adecuada como lo demuestra la pendiente de dispersión (Figura 5). pacientes clasificados como severos $(\mathrm{P}<0,0002)$.

tución en la que se realizó el estudio es mayor que en otros centros; lo que se explica porque se trata de un centro de tercer nivel y de referencia para patologías biliopancreáticas.

En nuestro medio la causa más común de la pancreatitis continúa siendo la biliar, al igual que en Inglaterra y el norte de Europa, a diferencia de lo que muestran los estudios norteamericanos donde es más frecuente la alcohólica. Cabe anotar que nuestra incidencia de pancreatitis aguda secundaria a colangiografía retrógrada endoscópica ha disminuido, lo que señala posiblemente el final de la curva de aprendizaje.

La mortalidad global se mantiene en 15 $\%$, teniendo en cuenta que la mayoría de nuestras pancreatitis son severas. Para la pancreatitis severa 25\%, lo que se correlaciona adecuadamente con la literatura mundial. ${ }^{18}$

Las complicaciones sistémicas de PA son iguales a las descritas en la literatura, con mayor incidencia en la falla orgánica múltiple, el síndrome de dificultad respiratoria del adulto y la insuficiencia renal aguda. ${ }^{19}$

De los pacientes estudiados, $60 \%$ fueron llevados a cirugía, de los cuales $36 \%$ fue de manera urgente, siendo la indicación más común el deterioro del APACHE II pese a una adecuada reanimación en la uni-

Los pacientes clasificados como severos únicamente por criterio tomográfico de necrosis, no mostraron una adecuada correlación entre la severidad y la evolución final; contrariamente a los que se clasificaron sólo por el cálculo del APACHE II (Tabla).

\section{Discusión}

El volumen de pacientes, si bien ha disminuido respecto a estudios previos ${ }^{17}$ en la misma institución, aún permite realizar trabajos en este campo de la cirugía. La severidad de la pancreatitis en la insti- dad de cuidados intensivos (UCI). Esta indicación, si se realiza en la primera semana de iniciado el cuadro agudo, es cada día más debatida. ${ }^{20}$

Cuando se clasifica la PA por el APACHE II, y se determina la presencia o no de necrosis con la TAC dinámica de abdomen, se encuentra una excelente correlación de la severidad con la necesidad de UCI, la morbilidad, la mortalidad y la necesidad de cirugía de urgencia, lo que corrobora los estudios de Balthazar, Ranson, McMahon, Marvin, Healt e Imrie. 


\section{Análisis estadístico}

Necrosis por TAC

C.N.

U.C.I.

Cirugía

Morbilidad

Mortalidad
$7 / 12$

$4 / 7$

$6 / 8$

$2 / 3$
$5 / 12$

$3 / 7$

$2 / 8$

$1 / 3$
S.N.

0.55

0.17

0.08

0.59
APACHE II

$>8$ $<8 \mathrm{P}$

2/12

0.002

$0 / 7$

$<0.0001$

$0 / 8$

$<0.0001$

$0 / 3$

Tabla

Si se clasifica por el APACHE II se mejora esta correlación $(\mathrm{P}<0.0003)$, contrario a lo sucedido cuando la severidad es determinada por la presencia o no de necrosis ( $\mathrm{P}>0.005)$. Lo que refleja que la tomografía dinámica realizada en las primeras 72 horas de iniciado el ataque no es buen marcador pronóstico para identificar aquellos pacientes que presentarán deterioro sistémico en las dos primeras semanas.

No se puede desconocer que la TAC dinámica es excelente elemento pronosticador de las complicaciones locales que generalmente aparecen desde la cuarta semana.

La tomografía dinámica tiene indicaciones precisas en la PA, sin embargo este trabajo pone en tela de juicio la necesidad de la tomografía en las primeras 72 horas sin importar el APACHE II y los criterios de Ranson.

\section{Conclusiones}

Si la tomografía dinámica, como lo indica este estudio, no prevé las complicaciones sistémicas tempranas, solo el cálculo del APACHE II y los criterios de Ranson serían útiles para clasificar la PA en severa tempranamente.

El único papel que juega la cirugía en la PA, en las primeras cuatro semanas, es controlar y resecar el te- jido pancreático necrótico infectado, puesto que han sido abandonados los conceptos de resecar el tejido pancreático inflamado como medida para disminuir la respuesta inflamatoria sistémica, ${ }^{21}$ o llevar a cirugía tempranamente a pacientes con necrosis estéril. ${ }^{22}$

La mortalidad de las dos primeras semanas es secundaria a las complicaciones sistémicas, generalmente por falla orgánica múltiple, mortalidad que es inmodificable por la cirugía. ${ }^{22}$ Después de la segunda semana la mortalidad está dada por la presencia de necrosis infectada.

Como se sabe, la necrosis pancreática se ve colonizada por bacterias solo en el $28 \%$ de los casos después de la segunda semana; por ello, no tiene objeto solicitar una tomografía antes de las primeras 72 horas para "ver si hay necrosis" así muestre un APACHE II superior a 8 o más de tres criterios de Ranson, puesto que la utilidad está dada por la identificación de necrosis infectada.

\section{Referencias}

1. Bradley LE III. A clinically based clasification system for acute pancreatitis. Summary of the Symposium on Acute Pancreatitis, Atlanta, GA. Arch Surg 1993;128: 586-590.

2. Schölmerich J, Messmann H. Serum markers for acute pancretitis - mirages or meaningful? Acute pancreatitis Blackwell Wissenschafts-Verlag GmbH. Berlin 1999.

3. White EM, Wittenberg J, Mueller PR, et al. Pancreatic necrosis: CT manifestations. Radiology 1986;158:343-46. 
4. Bradley EL, Frederick M, Ferguson C. Prediction of pancreatic necrosis by dynamic pancreatography. Ann Surg 1989;210:495-503.

5. Ranson JH, Balthazar E, Caccavale R, Cooper M. Computed tomography and the prediction of pancreatic abscess in pancreatitis. An Surg 1985; 201: 656-65.

6. Balthazar E, Robinsan D, Megibow A, Ranson JHC. Acute pancreatitis: Value of CT in establishing Prognosis. Radiology 1990; 174: 331-336.

7. London NJM, Leese T, Lavelle JM, West KP, et al. Rapid bolus contrast-enhanced dynamic computed tomography in acute pancreatitis: a prospective study. Br J Surg 1991; 78(18):1452-1456.

8. Ranson JH. Diagnostic standards for acute pancreatitis. World J Surg 1997;21(2):136-42.

9. Lucarotti ME, Virjee J, Anderson D. Patient selection and timing of dynamic computed tomography in acute pancretitis. Br J Surg 1993; 80(11):1393-1395.

10. Agarwal N, Pitchumoni CS. Assessment of severity in acyte pancreatitis. Am J Gastroenterol 1991; 86:1385-1391.

11. London NJM, Neoptolemos JP, Lavelle J et al. Contrastenhanced abdominal computed tomography scanning and prediction of severity of acute pancreatitis: a prospective study. Br J Surg 1989; 78: 268-272.

12. London NJM, Neoptolemos JP, Lavelle J, et al. Serial computed tomography scanning in acute pancreatitis: a prospective study. Gut 1989; 30: 397-403.
13. McMenamin D, Gates LK. A retrospective analysis of the effect of Contrast-Enhanced CT on the outcome of acute pancreatitis. Am J Gastroenterol 1996; 91(7):1384-1387.

14. Knaus WA, Draper EA, Wagner DP, Zimmerman JE. APACHE II: A severity of disease classification system. Crit Care Med 1985; 13: 818-829.

15. Larvin M, McMahon M. APACHE II score for assessment and monitoring of acute pancreatitis. Lancet 1989; 2: 201-204.

16. Wilson C, Heath DI, Imrie CW. Prediction of outcome in acute pancreatitis: a comparative study of APACHE II, clinical assessment and multiple factor scoring systems. Br J Surg 1990; 77:1260-1264.

17. Baron V, Gómez G, Riveros R. Pancreatitis aguda. Protocolo de manejo - Estudio prospectivo. Servicio de Cirugía, Hospital de San José, Hospital Occidente de Kennedy 1996.

18. Lankisch P, Burchard-Reckert S et al. Morbidity and Mortality in 602 patients with acute pancreatitis seen between the years 1980-1994. Z Gastroenterol 1996; 34: 371-377.

19. Thomson S, Hendry W, McFarlane G. Epidemiology and outcome of acute pancreatitis. Br J Surg 1987; 74: 398-401.

20. Russell R. Indications for Surgery: What has changed? Acute Pancreatitis; Blackwell Wissenschafts-Verlag GmbH. Berlin 1999.

21. Bradley EL III. Surgical indications and tecniques in necrotizing pancreatitis. Acute pancreatitis: diagnosis and therapy. Raven press Ltd., New York 1994.

22. Mier J, Leon E, Castillo A. Early versus late necrosectomy in severe necrotizing pancreatitis. Am J Surg 1997;173: 71-75.

\section{SOCIEDAD DE CIRUGÍA DE BOGOTÁ HOSPITAL DE SAN JOSÉ 1902 - 2001 HOSPITAL UNIVERSITARIO}

Atención integral del paciente, con énfasis en el manejo de alta complejidad, tecnología y excelente calidad de atención en cirugía y subespecialidades

Área clínica y subespecialidades

Pediatría y subespecialidades

- Servicios de apoyo diagnóstico: Resonancia nuclear magnética, Medicina nuclear, Tomografía axial computarizada, Banco de sangre, Laboratorio clínico y de inmunopatología 